\title{
Gastrointestinal stromal tumors treated at a Brazilian Cancer Center: evaluation of clinical, epidemiological, and therapeutic profiles
}

\author{
Erinaldo Rocha Paes Landim', Carolina de Barros Jaqueta ${ }^{1}$, Rubens Chojniak ${ }^{1,2}$, Felipe José Fernandez Coimbra ${ }^{3}$, \\ Celso Abdon Lopes de Mello ${ }^{4}$, Patricia Oriani ${ }^{1}$, Almir Galvão Vieira Bitencourt ${ }^{2 *}$ and Luana de Paula Silva Paes ${ }^{5}$
}

\begin{abstract}
Background: Gastrointestinal stromal tumors (GISTs) originate as precursor cells of the interstitial cells of Cajal in the myenteric plexus and generally have a mutation in the tyrosine kinase receptor, C-KIT (CD117). The objective is to evaluate the clinical, epidemiological, and therapeutic profiles of GIST cases available from a hospital specializing in cancer treatment.

Methods: A retrospective, longitudinal study of 85 GIST cases in a Cancer Center (São Paulo, Brazil) was conducted.

Results: The cases identified involved 40 men and 45 women and the average age at diagnosis was $55.7 \pm 14$. 8 years (median, 57). The symptoms present at diagnosis depended on the location and dimensions of each lesion. In 49 cases (57.6\%), the tumors had a gastric location and the mean dimensions were $7.2 \pm 2.3 \mathrm{~cm}$ (median, $3.4 \mathrm{~cm}$ ). Recurrent metastatic disease presented in 27 cases (with the liver mainly affected). Locoregional recurrence was detected in 16 cases. C-KIT was positive in 79/81 (97.5\%) of the cases examined. Most of the tumors were initially treated with surgery, while clinical treatment was applied to the recurrent cases. The overall survival rate was $76.4 \% 162$ months after diagnosis.

Conclusions: The GISTs examined most commonly originated in the stomach, while the liver was the main site affected by metastatic lesions. Most of the lesions appeared to be slow-growing neoplasms that were positive for C-KIT (CD117). Complications, as well as death, mostly affected the elderly patients that had comorbidities or more aggressive forms of the disease.
\end{abstract}

Keywords: Gastrointestinal stromal tumor, GIST, Stromal Cells, KIT

\section{Background}

Gastrointestinal stromal tumors (GISTs) are the most common malignant mesenchymal neoplasms of the gastrointestinal tract. They originate from precursor cells of the interstitial cells of Cajal in the myenteric plexus and they generally have a mutation in the receptor tyrosine kinase, C-KIT (CD117), or in a growth factor receptor derived from the platelet-derived growth factor receptor alpha (PDGFR- $\alpha)$. In recent years, GIST therapy has included tyrosine kinase inhibitors. In particular, the use

\footnotetext{
* Correspondence: almirgvb@yahoo.com.br

${ }^{2}$ Imaging Department, A.C. Camargo Cancer Center, São Paulo, Brazil

Full list of author information is available at the end of the article
}

of Imatinib mesylate and sunitinib malate have represented an advance in the treatment of GISTs [1].

The majority of data regarding GIST cases derive from Caucasians in industrialized countries. It is estimated that there are approximately 5000 to 6000 new cases annually in the United States, with an incidence ranging 6.5 to 14.5 cases per million between 1992 and 2000, with 54\% of the cases affecting men [2]. The increase in the number of GISTs diagnosed yearly is mainly due advances in our understanding of GIST histopathology; although, the incidence of GISTs may also be increasing. GISTs that are a few millimeters in diameter are common in adults and these lesions often contain an oncogenic mutation in KIT (CD117) or the PDGFR- $\alpha$ [3]. 
KIT is a receptor tyrosine kinase that has been well characterized and is frequently used for diagnosis of a GIST. Positivity for KIT also makes it possible to use a targeted therapy such as Imatinib. However, detection of KIT for a diagnosis has led to the false diagnosis of other mesenchymal tumors as GISTs. In addition, KIT concentrations can vary among tumors and the concentration of KIT in a tumor is not a predictor of its response to therapy with Imatinib [4]. Furthermore, while KIT positivity is a strong indicator for a diagnosis of GIST, this criterion may not be sufficient since metastatic melanoma, angiosarcoma (50\%), Ewing's sarcoma (50\%), childhood neuroblastoma (30\%), extramedullary myeloid tumors, seminoma, and small-cell pulmonary carcinomas are also positive for KIT [5]. Other markers that are frequently expressed by GISTs include: CD34 (70\%), actin $(30-40 \%)$, desmin $(<5 \%)$, and $\mathrm{S} 100$ proteins $(\sim 5 \%)$ [5]. Approximately 5\% of GISTs are negative for KIT expression [6]. Consequently, the diagnosis of KIT-negative GISTs remains a challenge and these cases are best handled by an expert pathologist, using additional tests such as PDGFR- $\alpha$ and DOG-1. A precise diagnosis is extremely important because some KIT-negative tumors are sensitive to Imatinib [6-11].

The aim of the present study was to evaluate the clinical, epidemiological and therapeutic aspects of GIST cases diagnosed at a cancer center in order to better understand the behavior and current management of this entity.

\section{Methods}

Initially, a search of the digital records system of a Cancer Center database was conducted with assistance from the information technology by searching for the keyword, "GIST". This search retrieved 97 records that were analyzed separately and spanned the time period of 2000 to 2014. Of these 97 cases, 91 received a diagnosis of GIST, while six had a differential diagnoses. Pathology diagnosis of GIST was an inclusion criterion for this study, therefore, the latter six cases were excluded. In further analysis, only 85 of the cases had unequivocal pathology diagnosis of GIST, while the other six cases did not. Medical records data from the 85 cases were utilized to conduct the retrospective longitudinal study and were analyzed using descriptive statistics.

Analyzed variables included gender, age, signs/symptoms, primary lesion site and size, immunohistochemistry (IHC) analysis, initial treatment, recurrence, retreatment, complications, follow-up and causes of death. Signs and symptoms were grouped as follows: abdominal pain (epigastralgia, general abdominal pain, pain in the right or left hypochondrium and anal pain), grastrointestinal (GI) bleeding (upper gastrointestinal bleeding, hematochezia, melena, hematemesis, enterorrhagia, anal bleeding), dyspepsia (nausea, bloating, heartburn, increase in abdominal volume), general unspecified symptoms (fever, fatigue and hyporexia) and less common symptoms. IHC analysis was performed in 81 patients and included KIT expression and other markers (e.g. CD34, DOG-1, Ki-67, AML, S100 protein, desmin, actin and vimentin). PDGFR- $\alpha$ mutation was evaluated in only nine patients. The cut-off used for Ki-67 expression was 10\%.

\section{Results}

Of the 85 GIST cases examined, 40 were male (48\%) and 45 female $(52 \%)$. The mean age at the time of diagnosis was $55.7 \pm 14.8$ years old (range, 94-19; median, 57). The symptoms presented at diagnosis included: abdominal pain $(n=26)$, GI bleeding $(n=15)$, dyspepsia $(n=13)$, weight loss $(n=6)$, diarrhea $(n=4)$, general unspecified symptoms $(n=3)$, acute bowel perforation $(n=2)$, constipation $(n=1)$, acute bowel obstruction $(n=1)$. In 17 cases, patients were asymptomatic and tumors were diagnosed by imaging exams (incidentalomas). For 10 cases, the symptoms at diagnosis were not recorded $(n=10)$.

The primary lesion sites (Fig. 1) included the stomach $(n=49)$, small intestine $(n=27)$, retroperitoneal space $(n=4)$, and rectum $(n=3)$. For two cases, the locations of the primary lesions were not stated. To evaluate the size of the primary lesions, the long axis of each lesion was measured. The mean long axis length was $7.2 \pm$ $2.3 \mathrm{~cm}$, with a median length of $3.4 \mathrm{~cm}$.

On IHC analysis, 79 out of 81 tumors (97.5\%) had positive KIT expression and two tumors were KITnegative GIST ( 1 had positive PDGFR- $\alpha$ and the other had inconclusive PDGFR- $\alpha$ with positive CD34 and DOG-1). PDGFR- $\alpha$ mutation was positive in seven out of nine patients tested (77.8\%). Other IHC markers tested as positive included: CD34 $(n=62 ; 72.9 \%)$; Ki-67 $(n=21 ; 24.7 \%) ;$ AML $(n=18 ; 21.2 \%) ;$ DOG- $1(n=8$; 9.4\%); S100 protein $(n=6 ; 7.1 \%)$; desmin $(n=4 ; 4.7 \%)$; actin $(n=3 ; 3.5 \%)$; and vimentin $(n=2 ; 2.4 \%)$.

Initial treatment for the cases examined most often involved surgery $(n=77)$, including 33 patients that underwent only surgery and 44 patients that also received clinical treatment. One patient performed only endoscopic resection and seven patients received only clinical treatment. For the 51 patients submitted to clinical treatment, 41 received Imatinib and ten received Sunitinib. When clinical treatment was selected as adjuvant, or neoadjuvant therapy, the patients received an initial dose of Imatinib at $400 \mathrm{mg} /$ day. Depending on each patient's need and tolerance, the dose was adjusted in some cases to $800 \mathrm{mg} /$ day. For cases involving resistance or intolerance, Sunitinib was administered at $50 \mathrm{mg} /$ day 


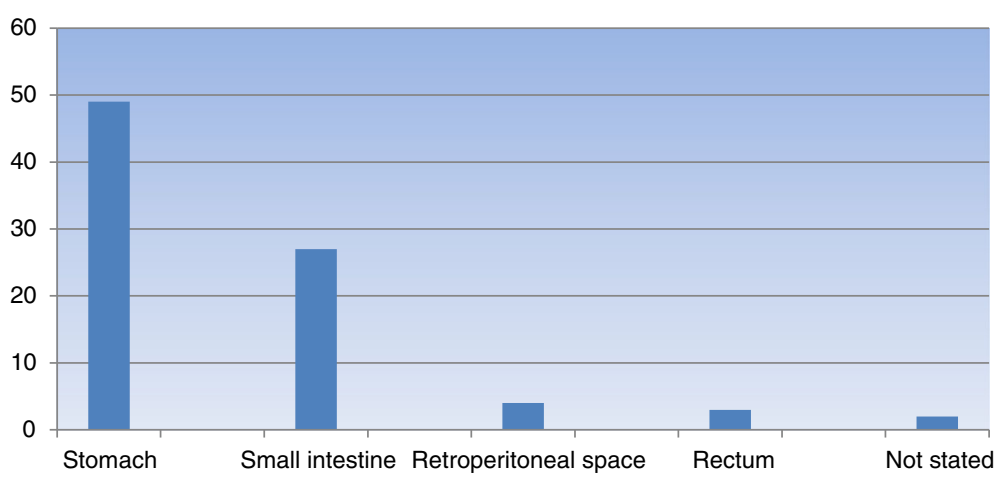

Fig. 1 Anatomical location distribution of the primary GISTS

or $37.5 \mathrm{mg} /$ day, respectively. Therapy selection was made based on each patient's status performance.

The main complications and morbidities that were initially observed or that arose during treatment were: symptomatic progression of metastatic disease $(n=4)$, thromboembolic events (e.g., deep vein thrombosis, pulmonary embolism) $(n=3)$, dyspeptic and dysphagia syndrome post-gastrectomy $(n=1)$, acute obstructive abdominal torsion $(n=1)$, intestinal perforation due to residual disease $(n=1)$, ischemic stroke $(n=1)$, epigastric hernia $(n=1)$, diarrhea $(n=3)$, hazy vision $(n=1)$, anastomotic esophagojejunal substenosis $(n=1)$, weight loss $(n=1)$, neutropenia $(n=1)$, abdominal wall abscess $(n=1)$, liver abscess $(n=1)$, pneumobilia $(n=1)$, edema and ascites $(n=3)$, non-neoplastic pleural effusion $(n=1)$, chronic abdominal pain $(n=1)$, anastomotic obstruction $(n=1)$, and bleeding by a chronic subdural hematoma $(n=1)$.

Recurrent disease presented as metastatic in 27 cases and locorregional in 16 cases. There were 38 intra- abdominal lesions and six extra-abdominal lesions that were located in the liver $(n=14)$, retroperitoneal space $(n=12)$, peritoneum $(n=7)$, spleen $(n=3)$, pancreas $(n=2)$, lungs $(n=2)$, uterus and fallopian tubes $(n=1)$, bone $(n=1)$, supraclavicular lymph node $(n=1)$, and inguinal lymph node $(n=1)$ (Fig. 2$)$.

Among the 44 cases that required retreatment due to either treatment resistance or relapse, clinical therapy was most commonly applied $(n=19)$, followed by surgery $(n=15)$ and a mixed therapy approach $(n$ $=10$ ). Regarding the clinical profile of the retreatment cases, Imatinib (400 mg/day) was administered in 13 cases, Imatinib (800 $\mathrm{mg} /$ day) was administered in three cases, and Sunitinib (50 mg/day) was administered in six cases.

A total of 61 cases were followed for 162 months, and the overall survival rate for these cases was $76,4 \%$ (Fig. 3). Death occurred in $14(23,6 \%)$ of these cases, being five in the first year after diagnosis, two in the period between 1 and 5 years, and seven after 5 years of

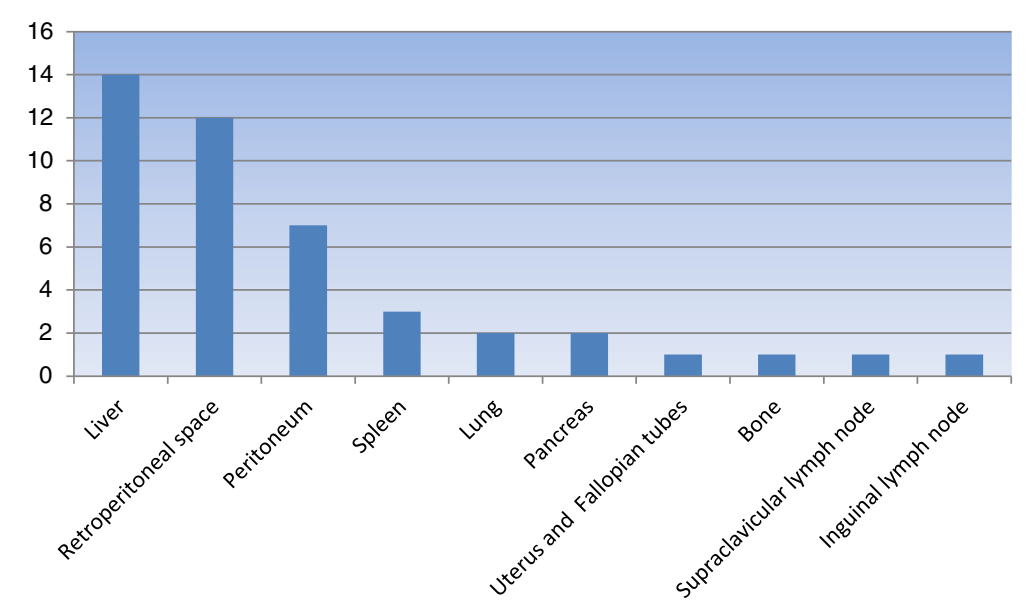

Fig. 2 Distribution of the metastatic disease sites 


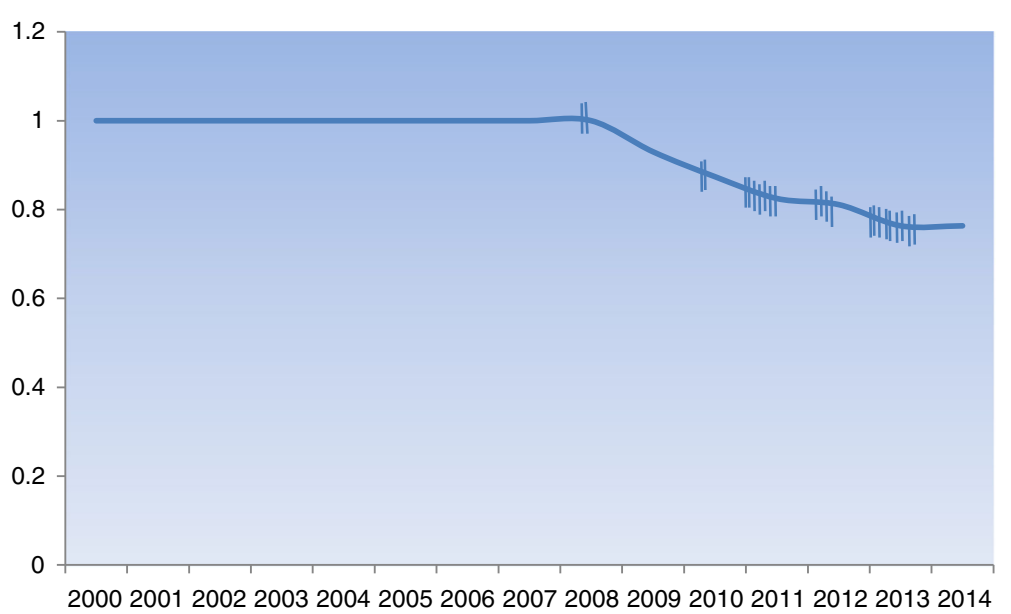

Fig. 3 Overall survival for 61 patients 162 months after diagnosis

diagnosis. The causes of death included: sepsis with a pulmonary focus $(n=4)$, pancytopenia $(n=1)$, unspecified sepsis $(n=3)$, complications from acute obstructive abdomen $(n=2)$, massive pulmonary embolism $(n=1)$, complication from another primary neoplasm $(n=1)$, complications from recurrent disease $(n=2)$. Overall, complications, as well as deaths, occurred mainly in the population of more elderly patients that had comorbidities or more aggressive forms of disease.

\section{Discussion}

Generally, GISTs are uncommon neoplasms. The present case series is important because of the number of patients included $(n=85)$ and because, in most cases, the GISTs were treated according to institutional protocols.

The cases examined in this study could not be categorized based on patient ethnicity because these data was not generally recorded. In Brazil, questions regarding ethnicity involve a great deal of subjectivity in terms of self-definition, and of intense miscegenation.

For the present cohort, the average age at diagnosis (mainly in the elderly), is consistent with international epidemiology data $[2,12,13]$. The most common sites for the primary lesions (stomach, 57.6\%) and metastatic lesions (liver, 32\%) examined are also consistent with that reported for Caucasian populations in developed countries [2]. However, the gender distribution of the GIST cases examined (males, 48\%; females $52 \%$ ) shows small difference from North American epidemiology statistics compiled between 1992 and 2000, where $54 \%$ of the cases affected men [2].

In general, surgery was the initial treatment selected for most of the cases examined. Furthermore, surgery played an important role also for the advanced disease. In 33 cases, surgery was sufficient for treatment.
For the high-risk and recurrent cases, systemic treatment with Imatinib or Sunitinib was more commonly selected as the treatment approach. In particular, treatment with Imatinib or Sunitinib was selected for cases with $\mathrm{Ki}-67$ present in more than $10 \%$ of the cancer cells (indicating a high mitotic index), as a neoadjuvant treatment for initially unresectable lesions, and for the treatment of recurrent disease or metastatic lesions. While morbidity was not absent, systemic therapy was well tolerated overall. The most frequent side effects were diarrhea, nausea, fatigue, muscle weakness, and abdominal pain [14]. The decisions to scale up the dosage of Imatinib from 400 to $800 \mathrm{mg} /$ day and to switch to Sunitinib (50 mg/day) in cases where evidence of clinical or immunological resistance or intolerance to the first-choice drug was observed and were consistent with the treatment plans employed by both European and American clinical trials $[15,16]$. Thus, patients are benefitting from the variety of drugs that are currently available for inhibiting tyrosine receptors.

Regarding the volumetric profile of the lesions treated, these data were consistent the presence of slow-growing neoplasms. The discrepancy observed between the median and average long axis data (e.g., $3.4 \mathrm{~cm}$ vs. $7.2 \mathrm{~cm}$ ) were due to a very large single lesion that measured $25 \mathrm{~cm}$ along its long axis. However, these data are consistent with the hypothesis that, in most cases, a GIST represents an indolent disease, and eventually a pseudodisease, with an absence of notable symptoms. In the present cohort, the symptoms at the time of diagnosis varied depending on the lesion site and the tumor's dimensions. It is worth emphasizing that there was a high number of GISTs diagnosed as an incidentaloma $(n=17)$, which naturally led us to consider that some of these cases might be indolent diseases. This 
finding consequently may raise a discussion on whether some patients could be elect for watchful waiting. Recent data suggest that the frequency of microscopic GISTs is higher than previously estimated. For example, in a Japanese study involving 100 resected stomach samples from patients with gastric cancer, 50 microscopic GISTs were detected in 35 patients and all of them were positive for KIT. Based on these results, the authors suggest that the low annual incidence of clinically detectable GISTs is due to a minority of GISTs that are potentially malignant $[17,18]$.

One aspect that was not addressed within the scope of the present study, although it is worth noting, is that new imaging technologies were important to monitor the success of using a pharmacological approach for tumor treatment. The imaging observation of GISTs behavior can provide a paradigm with which to observe tumor activity, response to therapy, and the tracking of solid lesions treated with targeted therapy. With computed tomography is an imaging method by which a reduction in attenuation (measured in Hounsfield units) can represent an increase in a lesion's liquid content and loss of cellularity or even cystic degeneration. Similarly, on magnetic resonance imaging, reduced tumor cellularity might be detected, especially when a diffusion technique is employed, since it allows a reduction in cellularity. Finally, tumor activity can be detected with positron emission tomography, and this approach can be used to detect the cytostatic effect of tyrosine kinase inhibitors. For example, lesions that are initially avid for FDG can show response by achieving reduction or absence of FDG concentration even without change in size [19-22] (Fig. 4).

The tumor marker profiling performed in the present study respected the definition of a neoplasm [1], and positivity for C-KIT (CD117) was detected in nearly all of the cases examined. The presence of PDGFR- $\alpha$ was not widely explored, although it showed positive expression in most cases that were tested. Positive expression of CD34 was also detected, while the other markers assayed were less prominent. Thus, additional studies are needed to establish the risk of recurrence in relation to Ki-67 expression.

Data regarding the biological behavior of GISTs following treatment with targeted molecular therapies provides valuable information regarding ongoing efforts to develop therapies that do not necessarily involve excision of a lesion. The advantages of a pharmacological approach are that it does not cause anatomofunctional damage and it prioritizes metabolic silencing, both of which lead to fewer side effects for patients. These advantages are clearly evident when comparing traditional chemotherapy to treatment with targeted molecular drugs such as monoclonal antibodies and small molecule inhibitors. Regarding the case series presented here, further clinical support for the use of tyrosine kinase inhibitors as a primary or adjuvant treatment for GISTs is demonstrated.

This study has the limitations of a retrospective study, based on information retrieved from nonstandard medical records. Because of that, we could not found standardized data related to imaging findings or the factors determining initial treatment in most cases and it was not possible to classify each patient in a relapse risk group according to a known system (e.g. Fletcher and/or Miettinen \& Lisota Risk Tables) for posterior analysis of outcome.

\section{Conclusions}

The GISTs examined most commonly originated in the stomach, while the liver was the main site affected by metastatic lesions. Most of the lesions appeared to be slowgrowing neoplasms, positive for C-KIT (CD117). Most patients were initially treated by surgery and were submitted to Imatinib either as adjuvant or neoadjuvant treatment. Complications, as well as death, mostly affected the elderly patients that had comorbidities or more aggressive forms of the disease. The case series presented here provide further clinical support for the use of tyrosine kinase inhibitors as a primary or adjuvant treatment for GISTs. Future prospective studies should focus on the relapse risk to optimize treatment and reduce complications and disease related death.

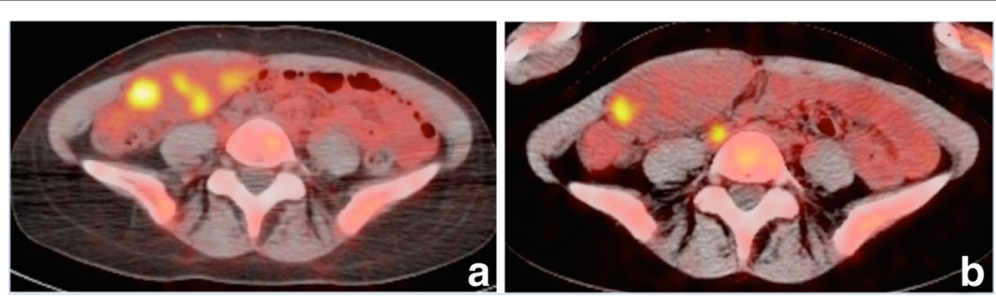

Fig. 4 Positron emission tomography-computed tomography (PET-CT) FDG: Pre (a) and post (b) Imatinib therapy. The abdominal mass presents decrease in FDG uptake inferring good response even without significant change in size 


\section{Abbreviations}

AML: Acute myeloid leukemia; C-KIT: Tyrosine kinase receptor; FDG: Fluorodeoxyglucose; GISTs: Gastrointestinal stromal tumors; PDGFRa: Platelet-derived growth receptor factor alpha; PET-CT: Positron emission tomography-computed tomography

\section{Acknowledgements}

None.

\section{Funding}

No funding received.

\section{Availability of data and materials}

The datasets analysed during the current study available from the corresponding author on reasonable request.

\section{Authors' contributions}

ERPL, CBJ, RC, FJFC and CALM designed the research; ERPL, CBJ, PO, AGVB and LPSP performed the research; ERPL, CBJ, RC, FJFC, CALM, PO and AGVB analyzed the data; and all authors wrote/revised the paper. All authors read and approved the final manuscript.

\section{Competing interests}

The authors declare that they have no competing interests. All procedures performed in these study were in accordance with the ethical standards of the institutional research committee and with the 1964 Helsinki declaration and its later amendments.

\section{Consent for publication}

Not applicable.

\section{Ethics approval and consent to participate}

This project received approval from the institution's Research Ethics Committee (no. 1931/14).

\section{Publisher's Note}

Springer Nature remains neutral with regard to jurisdictional claims in published maps and institutional affiliations.

\begin{abstract}
Author details
${ }^{1}$ Health Department, Universidade Nove de Julho (UNINOVE), São Paulo, Brazil. ${ }^{2}$ Imaging Department, A.C. Camargo Cancer Center, São Paulo, Brazil. ${ }^{3}$ Abdominal Surgery Department, A.C. Camargo Cancer Center, São Paulo, Brazil. ${ }^{4}$ Medical Oncology Department, A.C. Camargo Cancer Center, São Paulo, Brazil. ${ }^{5}$ Nursing Department, A.C. Camargo Cancer Center, São Paulo, Brazil.
\end{abstract}

Received: 28 September 2016 Accepted: 5 May 2017

Published online: 07 June 2017

\section{References}

1. Nishida T, Hirota S. Biological and clinical review of stromal tumors in the gastrointestinal tract. Histol Histopathol. 2000;15(4):1293-301.

2. Tran T, Davila JA, El-Serag HB. The epidemiology of malignant gastrointestinal stromal tumors: an analysis of 1,458 cases from 1992 to 2000. Am J Gastroenterol. 2005;100(1):162-8.

3. Steigen SE, Eide TJ. Trends in incidence and survival of mesenchymal neoplasm of the digestive tract within a defined population of northern Norway. APMIS. 2006;114(3):192-200.

4. Fletcher CD, Berman JJ, Corless C, Gorstein F, Lasota J, Longley BJ, et al. Diagnosis of gastrointestinal stromal tumors: A consensus approach. Hum Pathol. 2002;33(5):459-65.

5. Chirieac LR, Trent JC, Steinert DM, Choj H, Yang Y, Zhang J, et al. Correlation of immunophenotype with progression-free survival in patients with gastrointestinal stromal tumors treated with imatinib mesylate. Cancer. 2006:107(9):2237-44.

6. Debiec-Rychter M, Wasag B, Stul M, De wever I, Van Ossterom A, Hagemeijer A, et al. Gastrointestinal stromal tumours (GISTs) negative for KIT (CD117 antigen) immunoreactivity. J Pathol. 2004;202(4):430-8.
7. Medeiros F, Corless CL, Duensing A, Hornick JL, Oliveira AM, Heinrich MC, et al. KIT-negative gastrointestinal stromal tumors: proof of concept and therapeutic implications. Am J Surg Pathol. 2004;28(7):889-94.

8. Heinrich MC, Corless CL, Duensing A, McGreevey L, Chen CJ, Joseph N, et al. PDGFRA activating mutations in gastrointestinal stromal tumors. Science. 2003;299(5607):708-10.

9. Hirota S, Ohashi A, Nishida T, Isozaki K, Kinoshita K, Shinomura Y, et al. Gain-of-function mutations of platelet-derived growth factor receptor alpha gene in gastrointestinal stromal tumors. Gastroenterology. 2003;125(3):660-7.

10. Corless CL, Schroeder A, Griffith D, Town A, McGreevey L, Harrel P, et al. PDGFRA mutations in gastrointestinal stromal tumors: frequency, spectrum and in vitro sensitivity to imatinib. J Clin Oncol. 2005;23(23):5357-64.

11. West RB, Corless CL, Chen X, Rubin BP, Subramanian S, Montgomery K, et al. The novel marker, DOG1, is expressed ubiquitously in gastrointestinal stromal tumors irrespective of KIT or PDGFRA mutation status. Am J Pathol. 2004;165(1):107-13.

12. Nisson B, Bumming P, Meis-Kindblom JM, Odén A, Dortok A, Gustavsson B, et al. Gastrointestinal stromal tumors: the incidence, prevalence, clinical course, and prognostication in the preimatinib mesylate era-a population-based study in western Sweden. Cancer. 2005;103(4):821-9.

13. Tryggvason G, Gislason HG, Magnússon MK, Jónasson JG. Gastrointestinal stromal tumors in Iceland, 1990-2003: the icelandic GIST study, a population-based incidence and pathologic risk stratification study. Int J Cancer. 2005:117(2):289-93.

14. Demetri GD, Benjamin RS, Blanke CD, Blay J, Casali P, Choi H, et al. NCCN Task Force report management of patients with gastrointestinal stromal tumor (GIST)-update of the NCCN clinical practices guidelines. J Natl Compr Cancer Netw. 2007:5(Suppl 2):S1-S29.

15. Verweij J, Casali PG, Zalcberg J, LeCesne A, Reichardt P, Blay JY, et al. Progression-free survival in gastrointestinal stromal tumors with high-dose imatinib: randomised trial. Lancet. 2004:364:1127-34.

16. Blanke CD, Rankin C, Demetri GD, Ryan CW, von Mehren M, Benjamin RS, et al. Phase III randomized, intergroup trial assessing imatinib mesylate at two dose levels in patients with unressectable or metastatic gastrointestinal stromal tumors expressing the kit receptor tyrosine kinase: S0033. J Clin Oncol. 2008. doi:10.1200/JCO.2007.13.4452.

17. Kawanowa K, Sakuma Y, Sakuray S, Hishima T, Iwasaki Y, Saito K, et al. High incidence of microscopic gastrointestinal stromal tumors in the stomach. Hum Pathol. 2006:37(12):1527-35.

18. Agaimy A, Wunsch PH, Hofstaedter F, Blaszyk H, Rümmele P, Gaumann A, et al. Minute gastric sclerosing stromal tumors (GIST tumorlets) are common in adults and frequently show c-KIT mutations. Am J Surg Pathol. 2007;31:113-20.

19. Benjamin RS, Choj H, Macapinlac HA, Burgess MA, Patel SR, Chen LL, et al. We should desist using RECIST, at least in GIST. J Clin Oncol. 2007:25(13):1760-4.

20. Le Cesne A, Van Glabbeke M, Verweij J, Casali PG, Findlay M, Reichardt P, et al. Absence of progression as assessed by response evaluation criteria in solid tumors predicts survival in advanced Gl stromal tumors rreated with imatinib mesylate: the Intergroup EORTC-ISG-AGITG phase III trial. J Clin Oncol. 2009;27(24):3969-74.

21. Van den Abbeele AD, Badawi RD, Tetrault RJ, Cliche JP, Manola J, Spangler T, et al. FDG-PET as a surrogate marker for the response to Gleevec (imatinib mesylate) in patients with advanced gastrointestinal stromal tumors (GIST). J Nucl Med. 2003:44:77.

22. Van den Abbeele AD, Badawi RD. Use of positron emission tomography in oncology and its potential role to assess response to imatinib mesylate therapy in gastrointestinal stromal tumors (GISTs). Eur J Cancer. 2002:38(Suppl 5):S60-5. 